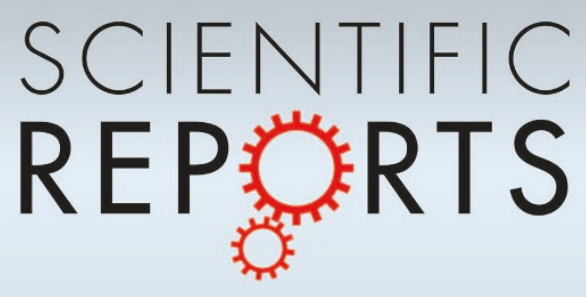

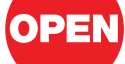

SUBJECT AREAS:

ELECTRONIC PROPERTIES

AND MATERIALS

SUPERCONDUCTING PROPERTIES

AND MATERIALS

CONDENSED-MATTER PHYSICS

MATERIALS SCIENCE

Received

21 January 2013

Accepted

15 May 2013

Published

31 May 2013

Correspondence and requests for materials should be addressed to

H.I. (h-iwasawa@ hiroshima-u.ac.jp) or Y.A. (y.aiura@aist.go.

ip)

\section{'True' bosonic coupling strength in strongly correlated superconductors}

\author{
Hideaki Iwasawa', Yoshiyuki Yoshida², Izumi Hase², Kenya Shimada', Hirofumi Namatame', \\ Masaki Taniguchi ${ }^{1,3} \&$ Yoshihiro Aiura ${ }^{2}$
}

\begin{abstract}
${ }^{1}$ Hiroshima Synchrotron Radiation Center, Hiroshima University, Higashi-Hiroshima, Hiroshima 739-0046, Japan, ${ }^{2}$ National Institute of Advanced Industrial Science and Technology, Tsukuba, Ibaraki 305-8568, Japan, ${ }^{3}$ Graduate School of Science, Hiroshima University, Higashi-Hiroshima, Hiroshima 739-8526, Japan.
\end{abstract}

Clarifying the coupling between electrons and bosonic excitations (phonons or magnetic fluctuations) that mediate the formation of Cooper pairs is pivotal to understand superconductivity. Such coupling effects are contained in the electron self-energy, which is experimentally accessible via angle-resolved photoemission spectroscopy (ARPES). However, in unconventional superconductors, identifying the nature of the electron-boson coupling remains elusive partly because of the significant band renormalization due to electron correlation. Until now, to quantify the electron-boson coupling, the self-energy is most often determined by assuming a phenomenological 'bare' band. Here, we demonstrate that the conventional procedure underestimates the electron-boson coupling depending on the electron-electron coupling, even if the self-energy appears to be self-consistent via the Kramers-Kronig relation. Our refined method explains well the electron-boson and electron-electron coupling strength in ruthenate superconductor $\mathrm{Sr}_{2} \mathrm{RuO}_{4}$, calling for a critical revision of the bosonic coupling strength from ARPES self-energy in strongly correlated electron systems.

T he many-body effects, the coupling between electrons and various excitations, are contained in the electron self-energy $\sum(\boldsymbol{k}, \omega)=\sum^{\prime}(\boldsymbol{k}, \omega)+i \sum^{\prime \prime}(\boldsymbol{k}, \omega)$, which is accessible via the spectral function $A(\boldsymbol{k}, \omega)$ measured by angle-resolved photoemission spectroscopy (ARPES) as

$$
A(\boldsymbol{k}, \omega)=-\frac{1}{\pi} \frac{\sum^{\prime \prime}(\boldsymbol{k}, \omega)}{\left[\omega-\varepsilon_{0}(\boldsymbol{k})-\sum^{\prime}(\boldsymbol{k}, \omega)\right]^{2}+\left[\sum^{\prime \prime}(\boldsymbol{k}, \omega)\right]^{2}}
$$

where $\varepsilon_{0}(\boldsymbol{k})$ is the non-interacting (bare) energy-band dispersion and the real and imaginary parts of the selfenergy, $\Sigma^{\prime}(\boldsymbol{k}, \omega)$ and $\Sigma^{\prime \prime}(\boldsymbol{k}, \omega)$ accounts for the energy renormalization and the lifetime broadening, respectively ${ }^{1}$. Assuming that the $\boldsymbol{k}$-dependence of the self-energy is negligible, $\sum(\boldsymbol{k}, \omega) \sim \sum(\omega)$, one can extract $\sum(\omega)$ and $\sum^{\prime \prime}(\omega)$ by analyzing the momentum distribution curves (MDCs) at constant energy $\omega ; \Sigma^{\prime}(\omega)=\omega-\omega_{0}(\boldsymbol{k})$ and $\sum^{\prime \prime}(\omega)=-v_{0} \Delta k$, where $v_{0}$ is the group velocity of the bare band $\left(\hbar^{-1} \partial \varepsilon_{0}(\boldsymbol{k}) / \partial \boldsymbol{k}\right)$ and $\Delta k$ is the MDC half width ${ }^{1}$. However, the derivation of the self-energy depends on the unfounded assumption of a bare band, which cannot be directly observed. To overcome this difficulty, the self-consistency of the self-energy via Kramers-Kronig relation is the most widely used criteria to extract the self-energy $y^{2,5-8}$. Moreover, iterative fitting algorithms for the determination of the bare band have also been developed based on the maximum entropy method $^{9-11}$ or the Kramers-Kronig relation ${ }^{12-14}$. In contrast, many previous ARPES experiments have regarded the bare band as a renormalized band due to the electron-electron coupling, which has enabled evaluation of the 'effective' bosonic self-energy and coupling strength ${ }^{2-8}$. However, the term 'effective' is often abbreviated or overlooked in many cases, although it has been proposed that the effective electron-boson coupling strength is smaller than the true coupling strength because of the electron-electron coupling ${ }^{15,16}$. There is a need, therefore, to reconsider and/or refine the most commonly used method for extracting the bosonic self-energy from ARPES data.

\section{Results}

We assume two main scattering channels, the electron-boson and electron-electron couplings. The energy-scales associated with these two are quite different: the electron-boson coupling dominates at low energy-scales (at most $\sim 100 \mathrm{meV}$ ), whereas the electron-electron coupling over a wide energy-scale (eV-order). The two are thus nearly independent in the scattering process and hence the Matthiessen's rule holds ${ }^{17}$. Then, the total self-energy and 


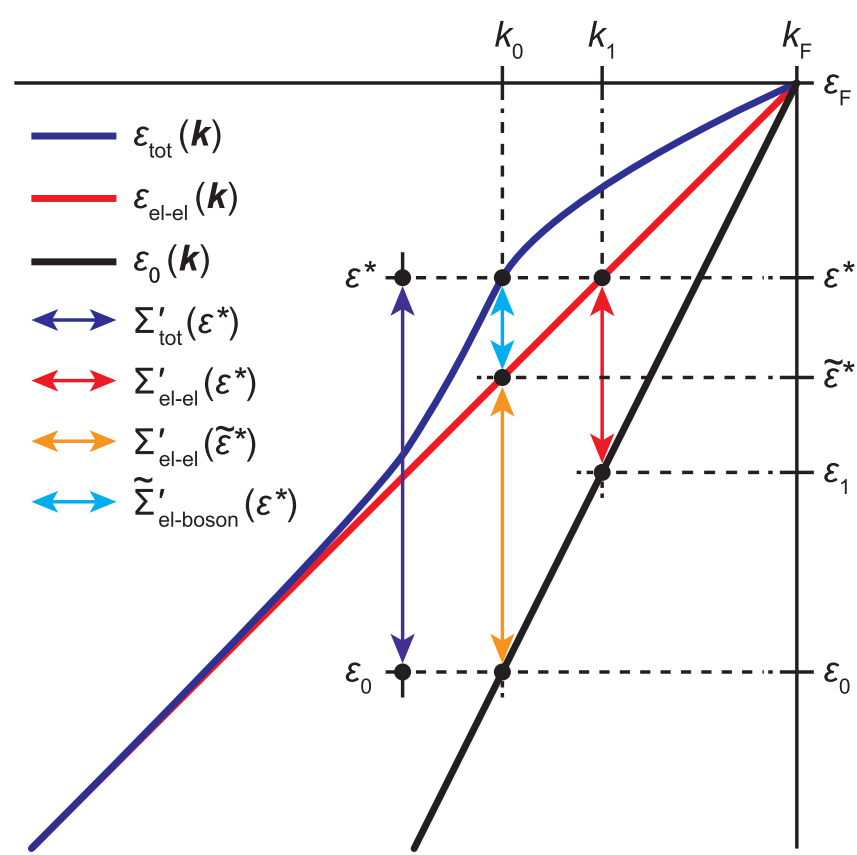

Figure $1 \mid$ Schematic illustration of the energy-band renormalization. The bare dispersion $\varepsilon_{0}(\boldsymbol{k})$ (black) is renormalized to $\varepsilon_{\mathrm{el}-\mathrm{el}}(\boldsymbol{k})$ (red) through the electron-electron coupling. Afterwards, $\varepsilon_{\mathrm{el}-\mathrm{el}}(\boldsymbol{k})$ is further renormalized to $\varepsilon_{\text {tot }}(\boldsymbol{k})$ (blue) through the electron-boson coupling. The arrows indicate the magnitude of the real-part of the self-energies.

coupling strength can be written as the summation of the contributions from these couplings; $\sum_{\text {tot }}(\omega)=\sum_{\text {el-boson }}(\omega)+\sum_{\text {el-el }}(\omega)$ and $\lambda_{\text {tot }}=\lambda_{\text {el-boson }}+\lambda_{\text {el-el }}$.

Figure 1 schematically illustrates the energy-band renormalizations: the bare dispersion $\varepsilon_{0}(\boldsymbol{k})$ (black) is renormalized due to the electron-electron coupling in the wide energy range resulting in $\varepsilon_{\text {el-el }}(\boldsymbol{k})(\mathrm{red})$, and further renormalized by the electron-boson coupling near the Fermi level resulting in $\varepsilon_{\text {tot }}(\boldsymbol{k})$ (blue). Note that $\varepsilon_{\text {el-el }}(\boldsymbol{k})$ corresponds to the 'bare' dispersion that has been extensively used so far. Here, we focus on the bosonic real-part of the self-energy, which can be defined at $k=k_{0}$ as

$$
\begin{gathered}
\sum_{\text {el-boson }}^{\prime}\left(\varepsilon^{*}\right)=\sum_{\text {tot }}^{\prime}\left(\varepsilon^{*}\right)-\sum_{\text {el-el }}^{\prime}\left(\varepsilon^{*}\right) \\
=\varepsilon_{1}-\varepsilon_{0}
\end{gathered}
$$

where we used relations $\sum_{\text {tot }}^{\prime}\left(\varepsilon^{*}\right)=\varepsilon^{*}-\varepsilon_{0}$ and $\sum_{\text {el-el }}^{\prime}\left(\varepsilon^{*}\right)=\varepsilon^{*}-\varepsilon_{1}$. Also, the group velocities of $\varepsilon_{0}(\boldsymbol{k})$ and $\varepsilon_{\mathrm{el}-\mathrm{el}}(\boldsymbol{k})$ are given by $v_{0}=$ $\left(\varepsilon_{1}-\varepsilon_{0}\right) /\left(k_{1}-k_{0}\right)$ and $v_{\text {el-el }}=\left(\varepsilon^{*}-\tilde{\varepsilon}^{*}\right) /\left(k_{1}-k_{0}\right)$, respectively, yielding $v_{0} / v_{\text {el-el }}=\left(\varepsilon_{1}-\varepsilon_{0}\right) /\left(\varepsilon^{*}-\tilde{\varepsilon}^{*}\right)=1+\lambda_{\text {el-el }}$ within the effective mass approximation. Now we can rewrite equation (2) as

$$
\begin{aligned}
\sum_{\text {el-boson }}^{\prime}\left(\varepsilon^{*}\right) & =\left(v_{0} / v_{\text {el-el }}\right)\left(\varepsilon^{*}-\tilde{\varepsilon}^{*}\right) \\
& =\left(1+\lambda_{\text {el-el }}\right) \widetilde{\sum}_{\text {el-boson }}^{\prime}\left(\varepsilon^{*}\right) .
\end{aligned}
$$

Here, we introduced $\widetilde{\Sigma}_{\text {el-boson }}^{\prime}\left(\varepsilon^{*}\right)=\varepsilon^{*}-\tilde{\varepsilon}^{*}$, which is equivalent to the 'effective' bosonic real part of the self-energy, namely, the energy shift due to the electron-boson coupling measured from $\tilde{\varepsilon}^{*}$, the renormalized energy arising from the electron-electron coupling. Consequently, the magnitude of the 'effective' bosonic real part of the self-energy is reduced by a factor of $\left(1+\lambda_{\text {el-el }}\right)$. Therefore, for the proper evaluation of the 'true' electron-boson coupling, especially for strongly correlated electron systems, it is indispensable to account for the electron-electron coupling.

Similarly, the coupling strength can be deduced by differentiating equation (3) as

$$
\lambda_{\text {el-boson }}=\left(1+\lambda_{\text {el-el }}\right) \widetilde{\lambda}_{\text {el-boson }}
$$

where $\lambda_{\text {el-boson }}=-\partial \sum_{\text {el-boson }}^{\prime} /\left.\partial \omega\right|_{\omega=0}$ and $\widetilde{\lambda}_{\text {el-boson }}=-\partial \widetilde{\Sigma}_{\text {el-boson }}^{\prime} /$ $\left.\partial \omega\right|_{\omega=0}$.

We emphasize the point that the problem with the conventional procedure is indeed simple and can be explicitly summarized by the following:

$$
\sum_{\text {tot }}^{\prime}\left(\varepsilon^{*}\right)=\widetilde{\Sigma}_{\text {el-boson }}^{\prime}\left(\varepsilon^{*}\right)+\sum_{\text {el-el }}^{\prime}\left(\tilde{\varepsilon}^{*}\right) .
$$

Comparing with equation (1), the energy reference point clearly differs for the electron-electron coupling, despite the summation of the real part of the self-energy having to be taken at the same energy a

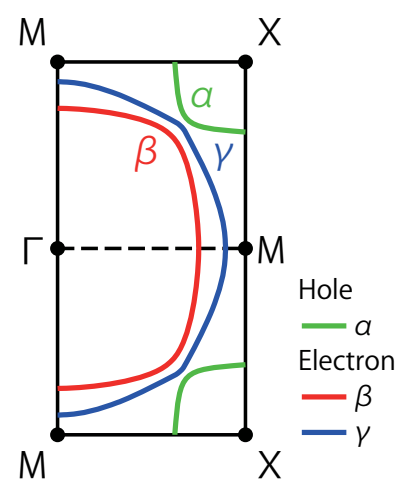

b

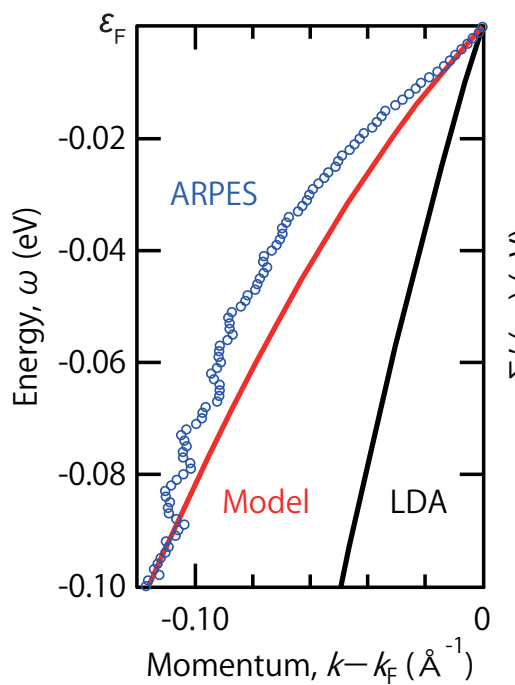

C

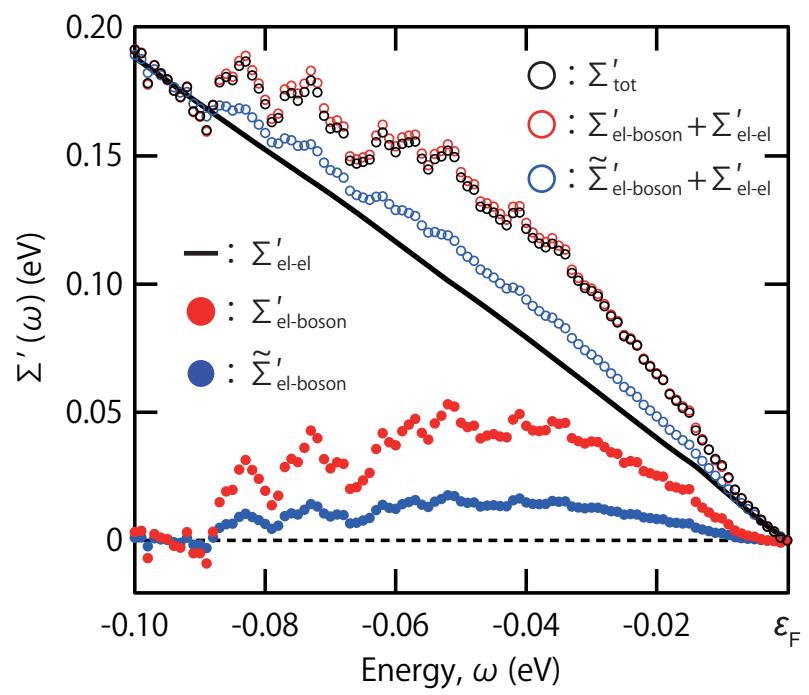

Figure $2 \mid$ Extraction of the 'true' and 'effective' bosonic real-part of the self-energy in $\mathrm{Sr}_{2} \mathrm{RuO}_{4}$. (a) Fermi surfaces of $\mathrm{Sr}_{2} \mathrm{RuO}_{4}$. (b) $\mathrm{MDC}$-derived energy-band dispersion (blue circles) for the $\gamma$-band, compared with the model dispersion (red) and LDA dispersion (black). The model dispersion includes band renormalization effects due to the electron-electron coupling as well as the spin-orbit coupling. (c) The real-part of the self-energies derived from the dispersions shown in $b$. 
Table 1 Quasiparticle parameters for the $\gamma$ band of $\mathrm{Sr}_{2} \mathrm{RuO}_{4}$ obtained from the present ARPES results compared with those from the LDA and Model calculations, and $\mathrm{dHvA}$ experiment ${ }^{18}$

\begin{tabular}{lcccc} 
& $k_{\mathrm{F}}\left(\AA^{-1}\right)$ & $v_{\mathrm{F}}(\mathrm{m} / \mathrm{s})$ & $m^{*}\left(m_{e}\right)^{a}$ & $m^{*} / m_{\mathrm{LDA}}^{*}{ }^{\mathrm{b}}$ \\
\hline LDA & 0.70 & $2.5 \times 10^{5}$ & 3.3 & 1.0 \\
Model & 0.72 & $8.7 \times 10^{4}$ & 9.6 & 2.9 \\
ARPES & 0.72 & $6.1 \times 10^{4}$ & 13.5 & 4.1 \\
dHvA & 0.75 & $5.5 \times 10^{4}$ & 16.0 & $4.9^{c}$
\end{tabular}

${ }^{\mathrm{a}} m^{*}=\hbar k_{F} / v_{F}$.

$\mathrm{b}_{m^{*}} / m_{\mathrm{LAA}}^{*}=\left(v_{\mathrm{F}}^{\mathrm{LDA}} / v_{\mathrm{F}}\right) /\left(k_{\mathrm{F}} / k_{\mathrm{F}}^{\mathrm{LDA}}\right)$.

cThe value is recalculated using present $m_{\mathrm{LDA}}$.

$\varepsilon^{*}$. As a result, the magnitude of the real part of the self-energy due to the electron-electron coupling is overestimated, producing subsequently an underestimated electron-boson coupling.

Similar arguments, described in Supplementary Information, also hold for the imaginary part of the self-energy. The 'true' and 'effective' bosonic real and imaginary parts of the self-energy only differs by factor $\left(1+\lambda_{\text {el-el }}\right)$, indicating that not only the 'true' but also the 'effective' bosonic self-energy should satisfy the Kramers-Kronig relation. This means that the self-consistency of the self-energy does not solely guarantee the validity of the bare band as well as the magnitude of the bosonic self-energy.

Based on the above methodology, we have examined the 'true' coupling strength of the electron-boson coupling in the unconventional ruthenate superconductor $\mathrm{Sr}_{2} \mathrm{RuO}_{4}$. The Fermi surface (FS) and low-lying electronic structure of $\mathrm{Sr}_{2} \mathrm{RuO}_{4}$ has been established by de Haas-van Alphen (dHvA), ARPES, and local-density approximation (LDA) band-structure calculation ${ }^{18,19}$. There are three FSs in $\mathrm{Sr}_{2} \mathrm{RuO}_{4}$ (see Fig. 2a): one hole-like $\alpha \mathrm{FS}$ around the $\mathrm{X}$ point, and two electron FSs $(\beta$ and $\gamma)$ around the $\Gamma$ point. The effective mass enhancements were also determined by dHvA experiments as $m^{*} /$ $m_{\mathrm{LDA}}(\alpha, \beta, \gamma)=(3.0,3.5,5.5)^{18}$, where $m^{*}$ and $m_{\mathrm{LDA}}$ is the effective mass and the LDA band mass, respectively. However, this poses a long-standing question as to why the largest mass enhancement emerges in the $\gamma$ FS formed by the widest $d_{x y}$ band $\left(W_{x y} \sim 3 \mathrm{eV}\right)$, and not in the $\alpha$ and $\beta$ FSs formed by the respective narrow $d_{y z}$ and $d_{z x}$ bands $\left(W_{y z, z x} \sim 1 \mathrm{eV}\right)$. We have therefore performed high-resolution ARPES measurements (see Methods) along the $\Gamma M$ line, where the $\gamma$ band can be almost solely observed, as was found in our previous polarization-dependent ARPES study ${ }^{20}$.

Figure $2 \mathrm{~b}$ shows the $\gamma$ band dispersion derived from ARPES (blue circles), model calculations (red) and LDA calculations (black). The model calculations include band renormalization effects due to the electron-electron coupling as well as the spin-orbit coupling, both of which were empirically evaluated based on ARPES data presented elsewhere $^{20-22}$ (see also Supplementary Information). The $\gamma$-quasiparticle parameter values, determined from these dispersions, are listed in Table 1; the values obtained from ARPES are found to be close to dHvA results ${ }^{18}$. The slight difference between the two results can be reasonably explained by instrument resolution, which distort the quasiparticle dispersion near the Fermi level (see Supplementary Information). With the available renormalized mass listed in Table 1, we can now disentangle the electron-boson and electron-electron

Table 2 | Electron-boson and electron-electron coupling strengths for the $\gamma$ band of $\mathrm{Sr}_{2} \mathrm{RuO}_{4}$, determined from the real part of the selfenergies

\begin{tabular}{lccc}
$\lambda_{\text {tot }}$ & $\lambda_{\text {el-el }}$ & $\lambda_{\text {el-boson }}$ & $\tilde{\lambda}_{\text {el-boson }}$ \\
\hline $3.1(3.1)$ & $2.0(1.9)$ & $1.1(1.2)$ & $0.36(0.41)$ \\
\hline
\end{tabular}

The coupling parameters deduced from the effective mass enhancement, $m^{*} / m_{\mathrm{LDA}}^{*}-1$, are also indicated in brackets. coupling strengths: $\lambda_{\text {tot }}=m_{\mathrm{ARPES}}^{*} / m_{\mathrm{LDA}}^{*}-1=3.1, \lambda_{\text {el-el }}=m_{\mathrm{Model}}^{*} /$ $m_{\mathrm{LDA}}^{*}-1=1.9$, and thus $\lambda_{\text {el-boson }}=\lambda_{\text {tot }}-\lambda_{\text {el-el }}=1.2$. The 'effective' bosonic coupling strength can be also calculated as $\tilde{\lambda}_{\text {el-boson }}=$ $m_{\text {ARPES }}^{*} / m_{\text {Model }}^{*}-1=0.41$. Note that one can confirm that equation (4) actually holds for these values.

The real part of the self-energies $\Sigma^{\prime}(\omega)$, given in Fig. $2 c$, can be extracted by subtracting two dispersions: $\sum_{\text {tot }}^{\prime}(\omega) \equiv \varepsilon_{\text {ARPES }}(\boldsymbol{k})-$ $\varepsilon_{\mathrm{LDA}}(\boldsymbol{k}), \quad \sum_{\text {el-el }}^{\prime}(\omega)=\varepsilon_{\text {Model }}(\boldsymbol{k})-\varepsilon_{\mathrm{LDA}}(\boldsymbol{k}), \quad$ and $\quad \widetilde{\Sigma}_{\text {el-boson }}^{\prime}(\omega)=$ $\varepsilon_{\text {ARPES }}(\boldsymbol{k})-\varepsilon_{\text {Model }}(\boldsymbol{k})$, which are marked with black open circles, a black solid line, and blue filled circles, respectively. We found that the summation of $\sum_{\text {el-el }}^{\prime}(\omega)$ and $\widetilde{\Sigma}_{\text {el-boson }}^{\prime}(\omega)$ (blue open circles) is completely different from the $\sum_{\text {tot }}^{\prime}(\omega)$, indicating that $\sum_{\text {tot }}^{\prime}(\omega) \neq$ $\sum_{\text {el-el }}^{\prime}(\omega)+\widetilde{\Sigma}_{\text {el-boson }}^{\prime}(\omega)$. By contrast, the summation of $\sum_{\text {el-el }}^{\prime}(\omega)$ and $\sum_{\text {el-boson }}^{\prime}(\omega)$ (red open circles) precisely coincides with $\sum_{\text {tot }}^{\prime}(\omega)$, thus satisfying equation (1). Here, $\sum_{\text {el-boson }}^{\prime}(\omega)$ (red filled circles) was derived from $\widetilde{\Sigma}_{\text {el-boson }}^{\prime}(\omega)$ multiplied by $\left(1+\lambda_{\text {el-el }}\right)$, where $\lambda_{\text {el-el }}=-\partial \sum_{\text {el-el }}^{\prime} /\left.\partial \omega\right|_{\omega=0}$. The coupling strengths, obtained from the derivative of the real part of the self-energy, are listed in Table 2; all are in good agreement with those obtained from the effective mass enhancement. Therefore, the present ARPES results clearly demonstrate the validity of our self-energy analysis.

\section{Discussion}

Contrary to the rather small 'effective' bosonic coupling strength $\tilde{\lambda}_{\text {el-boson }}=0.4$, the 'true' bosonic coupling strength is found to be as strong as $\lambda_{\text {el-boson }}=1.1-1.2$ in $\mathrm{Sr}_{2} \mathrm{RuO}_{4}$, which is a clear indication of strong coupling (e.g., see Ref. 23). Note that $\lambda_{\text {el-boson }}$ probably becomes $\sim 2.0$ if we consider instrument resolution (see Supplementary information). Such strong bosonic coupling can be expected to originate from the multiple bosonic couplings ${ }^{20}$, and its key ingredient could have particular relevance to the pairing mechanism, although at present we are unable to resolve this given the limited resolution as well as statistics.

Our results clearly outline the problem with the conventional selfenergy analysis, which often leads to an underestimation of the bosonic coupling strength by a factor of $\left(1+\lambda_{\text {el-el }}\right)$. The new insight we have presented here points toward a need for a critical revision of the bosonic coupling strength, that could probably rationalize the strong electron-boson coupling in strongly correlated superconductors ${ }^{15,24}$.

\section{Methods}

High-quality $\mathrm{Sr}_{2} \mathrm{RuO}_{4}$ single crystals were grown by the floating zone method with the self-flux technique ${ }^{25}$. Polarization-dependent ARPES measurements were performed at the linear undulator beamline (BL-1) of the Hiroshima Synchrotron Radiation Center using a high-resolution, hemispherical electron analyzer (R4000, VG-SCIENTA). All measurements were performed after cleaving the samples in situ under ultra-high-vacuum conditions $\left(\sim 2 \times 10^{-11}\right.$ Torr $)$ at temperatures lower than $10 \mathrm{~K}$. To eliminate the surface states of $\mathrm{Sr}_{2} \mathrm{RuO}_{4}$, the sample surface was aged, and then, ARPES data were collected at the photon energy of 42-eV with the

$s$-polarization geometry ${ }^{26}$. The polarization geometry, excitation energy, and location of the Brillouin zone were carefully determined from the ARPES data as described elsewhere ${ }^{20,21}$. The energy and angular resolutions were set to better than $10 \mathrm{meV}$ and $0.08^{\circ}$, respectively.

1. Damascelli, A., Hussain, Z. \& Shen, Z.-X. Angle-resolved photoemission studies of the cuprate superconductors. Rev. Mod. Phys. 75, 473 (2003).

2. Valla, T., Fedorov, A. V., Johnson, P. D. \& Hulbert, S. L. Many-Body Effects in Angle-Resolved Photoemission: Quasiparticle Energy and Lifetime of a Mo(110) Surface State. Phys. Rev. Lett. 83, 2085 (1999).

3. Lanzara, A. et al. Evidence for ubiquitous strong electron-phonon coupling in high-temperature superconductors. Nature 412, 510 (2001).

4. Johnson, P. D. et al. Doping and Temperature Dependence of the Mass Enhancement Observed in the Cuprate $\mathrm{Bi}_{2} \mathrm{Sr}_{2} \mathrm{CaCu}_{2} \mathrm{O}_{8+\delta}$. Phys. Rev. Lett. 87, 177007 (2001).

5. Reinert, F. et al. Electron-Phonon Coupling and its Evidence in the Photoemission Spectra of Lead. Phys. Rev. Lett. 91, 186406 (2003).

6. Kordyuk, A. A. et al. Bare electron dispersion from experiment: Self-consistent self-energy analysis of photoemission data. Phys. Rev. B 71, 214513 (2005).

7. Higashiguchi, Mitsuharu et al. Energy band and spin-dependent many-body interactions in ferromagnetic $\mathrm{Ni}(110)$ : A high-resolution angle-resolved photoemission study. Phys. Rev. B 72, 214438 (2005). 
8. Kordyuk, A. A. et al. Constituents of the Quasiparticle Spectrum Along the Nodal Direction of High- $T_{c}$ Cuprates. Phys. Rev. Lett. 97, 017002 (2006).

9. Shi, Junren et al. Direct Extraction of the Eliashberg Function for ElectronPhonon Coupling: A Case Study of Be(10і̄0). Phys. Rev. Lett. 92, 186401 (2004).

10. Zhou, X. J. et al. Multiple Bosonic Mode Coupling in the Electron Self-Energy of $\left(\mathrm{La}_{2-x} \mathrm{Sr}_{x}\right) \mathrm{CuO}_{4}$. Phys. Rev. Lett. 95, 117001 (2005)

11. Zhao, Lin et al. Quantitative determination of Eliashberg function and evidence of strong electron coupling with multiple phonon modes in heavily overdoped $(\mathrm{Bi}, \mathrm{Pb})_{2} \mathrm{Sr}_{2} \mathrm{CuO}_{6+\delta}$. Phys. Rev. B 83, 184515 (2011).

12. Veenstra, C. N., Goodvin, G. L., Berciu, M. \& Damascelli, A. Elusive electronphonon coupling in quantitative analyses of the spectral function. Phys. Rev. B 82, 012504 (2010)

13. Veenstra, C. N., Goodvin, G. L., Berciu, M. \& Damascelli, A. A. Spectral function tour of electron-phonon coupling outside the Migdal limit. Phys. Rev. B 84, 085126 (2011).

14. Pletikosić, I., Kralj, M., Milun, M. \& Pervan, P. Finding the bare band: Electron coupling to two phonon modes in potassium-doped graphene on $\operatorname{Ir}(111)$. Phys. Rev. B 85, 155447 (2012).

15. Kulić Miodrag, L. \& Dolgov Oleg, V. Angle-resolved photoemission spectra of $\mathrm{Bi}_{2} \mathrm{Sr}_{2} \mathrm{CaCu}_{2} \mathrm{O}_{8}$ show a Coulomb coupling $\approx 1$ and an electron-phonon coupling of 2-3. Phys. Rev. B 76, 132511 (2007).

16. Kordyuk, A. A. et al. Angle-resolved photoemission spectroscopy of superconducting LiFeAs: Evidence for strong electron-phonon coupling. Phys. Rev. B 83, 134513 (2011).

17. Ashcroft, N. W. \& Mermin, N. D. Solid State Physics (Saunders College, Philadelphia, 1976).

18. Mackenzie, A. P. \& Maeno, Y. The superconductivity of $\mathrm{Sr}_{2} \mathrm{RuO}_{4}$ and the physics of spin-triplet pairing. Rev. Mod. Phys. 75, 657 (2003).

19. Maeno, Yoshiteru et al. Evaluation of Spin-Triplet Superconductivity in $\mathrm{Sr}_{2} \mathrm{RuO}_{4}$ J. Phys. Soc. Jpn. 81, 011009 (2012).

20. Iwasawa, H. et al. Interplay among Coulomb Interaction, Spin-Orbit Interaction, and Multiple Electron-Boson Interactions in $\mathrm{Sr}_{2} \mathrm{RuO}_{4}$. Phys. Rev. Lett. 105, 226406 (2010).

21. Iwasawa, H. et al. High-Energy Anomaly in the Band Dispersion of the Ruthenate Superconductor. Phys. Rev. Lett. 109, 066404 (2012).

22. Aiura, Y. et al. Enhancement of Hybridization between Two- and OneDimensional Bands due to Coulomb and Spin-Orbit Interactions in $\mathrm{Sr}_{2} \mathrm{RuO}_{4}$. J. Phys. Soc. Jpn. 79, 123702 (1998)
23. Carbotte, J. P. Properties of boson-exchange superconductors. Rev. Mod. Phys. 62 , 1027 (1990).

24. Ruiz, H. S. \& Badia-Majós, A. Nature of the nodal kink in angle-resolved photoemission spectra of cuprate superconductors. Phys. Rev. B 79, 054528 (2009).

25. Yoshida, Y. et al. Fermi Surface and Yamaji Effect in $\mathrm{Sr}_{2} \mathrm{RuO}_{4}$. J. Phys. Soc. Jpn. 67, 1677 (1998).

26. Iwasawa, H. et al. Orbital selectivity of the kink in the dispersion of $\mathrm{Sr}_{2} \mathrm{RuO}_{4}$. Phys. Rev B 72, 104514 (2005).

\section{Acknowledgements}

We thank H. Hayashi, J. Jiang for their assistance in the ARPES experiments. This work was supported by KAK-ENHI (22740233). Synchrotron radiation experiments were performed under the approval from Hiroshima Synchrotron Radiation Center (Proposal Nos. 11-A-8 and 11-A-9).

\section{Author contributions}

H.I. and Y.A. designed and performed the ARPES experiment. H.I. performed the data analysis. Y.Y. grew high-quality single crystals. I.H. performed the LDA calculations. H.I. and Y.A. wrote the manuscript with input from K.S. and all other coauthors.

\section{Additional information}

Supplementary information accompanies this paper at http://www.nature.com/ scientificreports

Competing financial interests: The authors declare no competing financial interests.

License: This work is licensed under a Creative Commons

Attribution-NonCommercial-NoDerivs 3.0 Unported License. To view a copy of this license, visit http://creativecommons.org/licenses/by-nc-nd/3.0/

How to cite this article: Iwasawa, H. et al. 'True' bosonic coupling strength in strongly correlated superconductors. Sci. Rep. 3, 1930; DOI:10.1038/srep01930 (2013). 